Neural, Parallel, and Scientific Computations 29 (2021), No.1, 55 - 67

\title{
SPECTRUM RESOURCE ALLOCATION TO MAXIMIZE PERFORMANCE IN MOBILE D2D SYSTEMS
}

\section{STEVEN Y. M. CHEN}

Faculty of Automation, Guangdong University of Petrochemical Technology(GDUPT), China Email: chenyeeming@gmail.com

\begin{abstract}
Spectrum resource allocation is one of the promising technologies for improving service quality and data rate of mobile users. Even though spectrum resource can provide improved home coverage and throughput for indoor users, it causes interference to D2D( device to device) users when spectrum resource uses the same frequency band of cell. To reduce the interference between existing D2D and spectrum resource, it is needed to analyze the characteristic of cell and spectrum resource considering various interference scenarios. In this paper, we develop an analytical model of spectrum resource allocation based on D2D spectrum resource network. Further, employs genetic algorithm to dynamic channel allocation to maximize signal-tointerference-plus-noise ratio (SINR) in a near-optimal fashion.
\end{abstract}

Keywords: D2D(Device to Device) , SINR(signal-to-interference-plus-noise ratio) , Genetic algorithm. Interference.

\section{INTRODUCTION}

Spectrum resources are capable of providing services in shadowed areas of the device to device (D2D) cell coverage enhancement and can relieve traffic from the cell networks, reduce infrastructure costs for the network operators, provide high-data-rate services to the users in a costeffective manner and at the same time, to enhance network capacity. In this regard, spectrum resources are considered as a promising option for the home base stations to improve the cell coverage, especially in the interiors of houses and buildings and to provide ubiquitous high speed connectivity to the end users or User Equipments (UEs). Spectrum resources or Cell Access Points (CAPs) are small, short-ranged (10 30 m) low powered (10 100 mW) access points developed to provide cost-effective and high-bandwidth services[1]. One of the main advantages of spectrum resource deployment is the improvement of indoor coverage where mobile base station (referred to as MBS) signal is weak. Spectrum resources provide high data rate and improved quality-ofservice (QoS) to the subscribers. It also lengthens the battery life of the mobile phones since the mobile phones do not need to communicate with a distant cell base station. Spectrum resources

Received January 20, 2021 www.dynamicpublishers.org; https://doi.org/10.46719/npsc20202914
1061-5369 \$15.00 @ Dynamic Publishers, Inc. www.dynamicpublishers.com; 
can easily be deployed by the end users in indoor environments on a "plug-and-play" basis. As spectrum resource networks are customer-deployed without proper network planning, their interference environment tends to be much more complicated than the traditional cellular networks. Thus, interference problems in spectrum resource networks cannot be handled by existing schemes typically used for cell deployments [2]. In addition to the increased user throughput from short ranges, the smaller size of spectrum resources increases the system capacity by enabling spatial reuse. This allows broadband access service providers to (1) improve coverage and service quality, (2) effectively balance load by offloading traffic from cell to spectrum resources, and (3) reduce operational expenses and subscriber hand off.

The scope of this paper is hence to examine how users in D2D networks with spectrum resource allocation can share the available radio resources efficiently in order to mitigate cochannel interference and thus enhance the signal-to-interference-plus-noise ratio (SINR) of the networks. This article also proposed genetic algorithm(GA) heuristic approach on a prototype D2D spectrum resource network testbed and show that it assigns channels to interfering spectrum resources in a near-optimal fashion.

The rest of this paper is organized as follows. Section 2 provides the background and related D2D spectrum resource work. Section 3 describes the system model and path loss models. Spectrum resource allocation using genetic algorithm presented in Section 4. In Section 5, a prototype D2D spectrum resource network testbed and show its simulation results. Finally, we conclude in Section 6.

\section{BACKGROUND AND RELATED WORK}

In this section, we describe relevant related work. We then provide a brief background on D2D spectrum resource systems.

Mobile D2D (IEEE 802.16e) based on IEEE 802.16e standard has been commercialized in 2006 with rapid growth of various multimedia applications [3,4]. In current mobile communication systems, the signal strength transmitted from base station (BS) can be very low in indoor environments because the signal strength may be severely attenuated when it penetrates the obstacles such as walls. Thus, providing high data rate services to indoor users is difficult only in mobile D2D systems.

Spectrum resource is connected to IP based backhaul such as digital subscriber line (DSL) or cable modem, which provides lower cost than wireless mobile communication systems. Spectrum resources can achieve a high signal-to-interference-plus-noise ratio (SINR) with low 
transmit power because the distance between transmitter and receiver is short. In addition, the operators can save costs when spectrum resource accommodates traffic concentrated to cell of current mobile communication. Although spectrum resource has many advantages in indoor environments, some technical issues, such as spectrum assignment of spectrum resource, access policy, network synchronization, handover, self-optimization, and self-configuration, should be solved to be effectively deployed in existing mobile D2D networks [5]. Especially, channel assignment for avoiding interference between cell and spectrum resource is an important issue since interference may causes severe degradation of throughput and service quality [6].

When spectrum resources operate in the same spectrum in mobile D2D systems, the characteristic of interference depends on the method for channel assignment. Channel assignment method for cell and spectrum resource is classified into orthogonal and common channel assignment. Orthogonal channel assignment does not cause co-channel interference between the cell and spectrum resource because the spectrum resources use different channels with cell. However, orthogonal channel assignment has a low spectrum efficiency because frequency resources for the cell and spectrum resource are different. On the other hand, common channel assignment has a high spectrum efficiency because cell and spectrum resource can use all of the spectrum. However, there exists a problem of co-channel interference in common channel assignment method. When spectrum resources are deployed in a cell, we should also consider cochannel interference among spectrum resources. Interferences are caused by neighbouring spectrum resource BS and spectrum resource UE. Especially, when the distance between spectrum resources is short, the impact of interference from neighbouring spectrum resource may be high. That is, co-channel interference between spectrum resources can be a serious problem when a lot of spectrum resources are concentrated in a small area.

\section{D2D SPECTRUM RESOURCES SYSTEM MODEL}

To analyse the performance when spectrum resources are deployed in mobile D2D systems, we consider an existing D2D and spectrum resource users as shown in Figure 1. In this scenario, spectrum resources are deployed in the centre cell 1. The attenuation of transmitted signal, called path loss, can differ according to channel environment (e.g., indoor or outdoor). As path loss models, we apply Wireless World Initiative New Radio (WINNER) II model to evaluate the throughput [7]. 


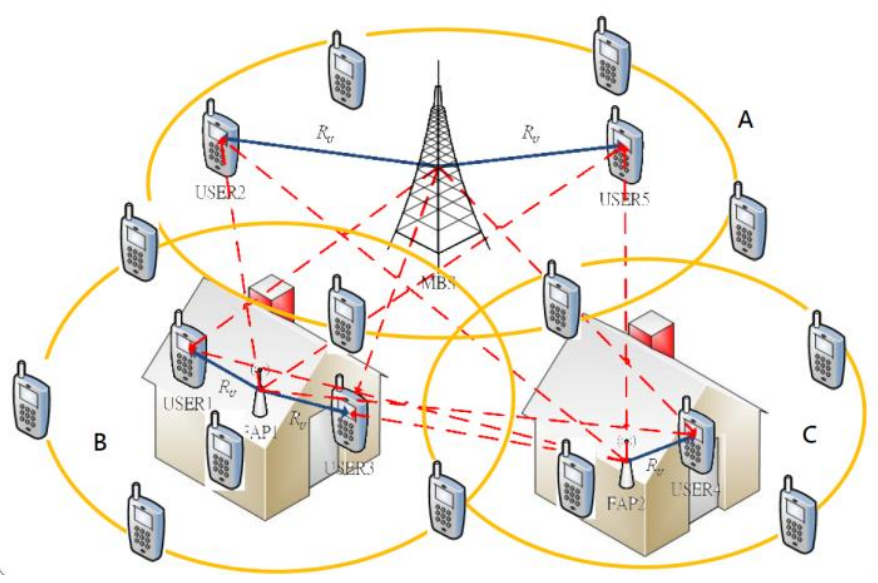

\section{Figure 1 The interference Scenarios between Existing D2D and Spectrum Resource Operations}

Path loss models for the various WINNER scenarios are typically of the form in equation 1, where $d$ is the distance between the transmitter and the receiver in [m], $f e$ is the system frequency in [GHz], the fitting parameter $A$ includes the path loss exponent, parameter $B$ is the constant term, parameter $C$ describes the path loss frequency dependence, and $X$ is an optional, environment specific term.

$$
P L(d)=A \cdot \log _{10}(d)+B+C \cdot \log _{10}\left(\frac{f e}{5}\right)+X
$$

In order to have a look at the different interference situations, an easy and intuitive idea would be to estimate the interference knowing the interferer path loss model for each scenario.

$\mathrm{B}, \mathrm{C}$ cases: indoor-to-indoor interference.

A-B, A-C: outdoor-to-indoor interference.

B-A, C-A cases: indoor-to-outdoor interference.

B-C case: indoor-to-outdoor-to-indoor interference.

To calculate the path loss between D2D MBS and spectrum resource UEs, we use non-line of sight (NLOS) outdoor propagation model. Also, we use NLOS indoor propagation model for calculating path loss between MBS and spectrum resources. Path loss of the indoor and outdoor signal is given as

$$
P L_{i n \rightarrow i n}(d)=36.8 \cdot \log _{10}(d)+37.78
$$




$$
\begin{aligned}
& P L_{\text {out } \rightarrow \text { in }}\left(d_{\text {out }}, d_{\text {in }}\right)=P L_{b}^{\text {out }}+0.5 \cdot d_{\text {in }}+29 \\
& P L_{\text {in } \rightarrow \text { out }}\left(d_{\text {in }}, d_{\text {out }}\right)=P L_{b}^{\text {in }}+0.5 \cdot d_{\text {in }}+29
\end{aligned}
$$

Where,

$$
\begin{aligned}
& P L_{b}^{\text {in }}=P L\left(d_{\text {in }}, d_{\text {out }}\right) \\
& P L_{b}^{\text {out }}=P L\left(d_{\text {out }}, d_{\text {in }}\right)
\end{aligned}
$$

and

$$
\begin{aligned}
& \operatorname{PL}\left(d_{1}, d_{2}\right)=22.7 \cdot \log _{10}\left(d_{1}\right) \\
& -12.5 \cdot\left\{\max \left(2.8-0.0024 \cdot d_{1}, 1.84\right)\right\} \\
& +10 \cdot\left\{\max \left(2.8-0.0024 \cdot d_{1}, 1.84\right)\right\}+19.1
\end{aligned}
$$

The received signal to interference ratio (SIR) is defined as the ratio of a signal power to the interference power. Let $\mathrm{p}_{\mathrm{s}}^{\mathrm{r}}$ and $\mathrm{I}_{\mathrm{s}}^{\mathrm{r}}$ be strengths of received signal and interference from sender $\mathrm{s}$ to receiver $\mathrm{r}$, and $\mathrm{M}$ and $\mathrm{F}$ be the number of cells and spectrum resources. $\mathrm{N}$ is white noise. SINR of user $\mathrm{n}$ in the downlink at MBS ${ }^{\mathrm{i}}$ is:

$$
\operatorname{SINR}_{M B S^{i}}^{\mathrm{n}}=\frac{\mathrm{P}_{M B S^{\mathrm{i}}}^{\mathrm{n}}}{\sum_{\mathrm{j}=1}^{\mathrm{F}} \mathrm{I}_{\mathrm{FAP}}^{\mathrm{n}}+\sum_{\mathrm{k}=1, \mathrm{k} \neq \mathrm{i}}^{\mathrm{M}} \mathrm{I}_{M A B^{\mathrm{k}}}^{\mathrm{n}}+\mathrm{N}}
$$

SINR of user $\mathrm{n}$ in the uplink at FAP ${ }^{\mathrm{i}}$ is:

$$
\operatorname{SINR}_{\text {user }^{\mathrm{i}}}^{\mathrm{n}}=\frac{\mathrm{P}_{\text {user }}^{\mathrm{n}}}{\sum_{\mathrm{j}=1, \mathrm{j} \neq \mathrm{i}}^{\mathrm{F}} \mathrm{I}_{\text {user }^{\mathrm{i}}}^{\mathrm{i}}+\sum_{\mathrm{k}=1}^{\mathrm{M}} \mathrm{I}_{\mathrm{FAP}}^{\mathrm{n}}+\mathrm{N}}
$$

The received SINR is used to calculate channel capacity with the Shannon-Hartley theorem. Channel capacity $\mathrm{C}$ is calculated as follows.

$$
\mathrm{C}=\mathrm{BW} \times \log _{2}(1+\mathrm{SINR})
$$

where $\mathrm{BW}$ is channel bandwidth in $\mathrm{Hz}$.

\section{SPECTRUM RESOURCE ALLOCATION USING GENETIC ALGORITHM}

Genetic algorithms are widely used stochastic search algorithm to find solution of complex problems [8]. A general GA uses selection, crossover and mutation operations to generate a new population with better fitness than actual population similarly to natural selection and sexual 
recombination. Generally, a population is a set of individual and an individual consists of genes. The so called elite individuals are the individuals in a population with best fitness scores. Parents are individuals of the actual population whereas children are the individuals of the new generated population. The evolution of the population takes place following the general GA principles through tournament selection, crossover and mutation. In addition, other genetic operators and techniques can be used to improve the performance of the GA, such as Elitism, it is implemented so that the best solution of every generation is copied to the next so that the possibility of its destruction through a genetic operator is eliminated. The flow chart of Figure 2 describes the main steps of the GA procedure. The contents of the blocks in the flow chart are explained in greater details in the next paragraphs.

\section{1)Initial solution}

As it can be seen from the Figure 2, an initial population is randomly generated. The population size can be designed by the user. The population is formed so that there are 12 individuals that are represented as vectors. The length of each of the individuals is the same as the number of FAPs connected to the network. The $\mathrm{i}$-th value in the vector gives the frequency channel that MBS is using

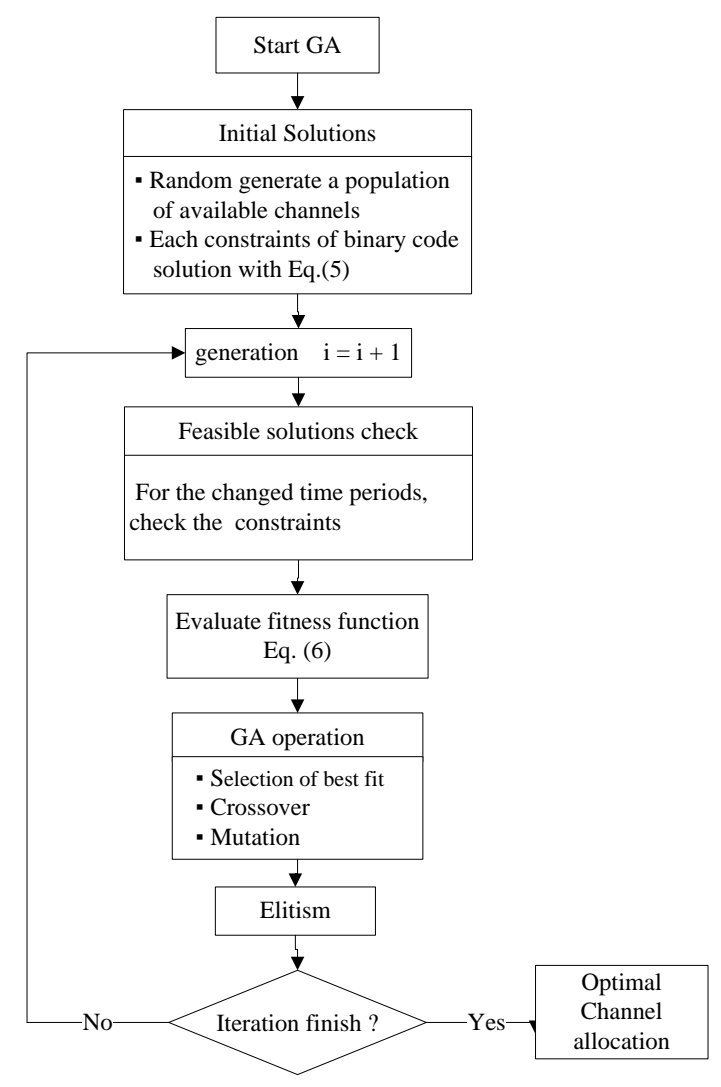




\section{2) Evaluation}

A D2D spectrum resource channel allocation has basically two characteristics. Firstly, the average signal-to-interference-and-noise ratio, SINR, that FAPs receive must be as high as possible. This reflects the overall performance of the network. On the other hand, the minimum SINR requirement for all the users must be met. The fitness function in this case has the following form:

$$
\text { fitness }=\text { SINR }+ \text { penalty }+ \text { jitter }
$$

where, SINR is derived from (3) and (4), penalty decreases the fitness if some FAPs experience a SINR that is lower than $9 \mathrm{dBm}$ (In this case the minimum requirement is set at $\mathrm{SINR}=9 \mathrm{~dB}$.), and jitter adds randomness.

\section{3)Selection}

Evaluation of the fitness criterion to choose which individuals from a population will go on to reproduce. Some general methods used are Roulette Wheel Selection and Tournament Selection. For this purpose, the chromosomes can be arranged in their increasing order of fitness values and the first half can be selected.

\section{4)Crossover}

Once a portion of the population has been selected, the number of chromosomes in the initial population decrements. But the population size must be maintained throughout. For this purpose, new chromosomes have to be generated from the existing ones. This is done with the help of two functions: crossover and mutation. Crossover is the process where two chromosomes are combined to form two new chromosomes. The strings that are selected from the selected population for this purpose are called parent chromosomes. The offsprings produced are called child chromosomes. Crossover can be either one-point or two-point crossover. Two parents and a random crossover point, cross point, is selected. A general algorithm for one-point crossover can be shown as:

function [child1, child2]=crossover(parent1,parent $2, p c)$;

if $($ rand $<p c)$

cross_point $=\operatorname{round}\left(\right.$ rand $^{*}($ stringlength -2$\left.)\right)+1$;

child $1=[$ parent $1(:, 1:$ cross_point $) \quad$ parent $2(:$, cross_point $+1:$ stringlength $)]$; 


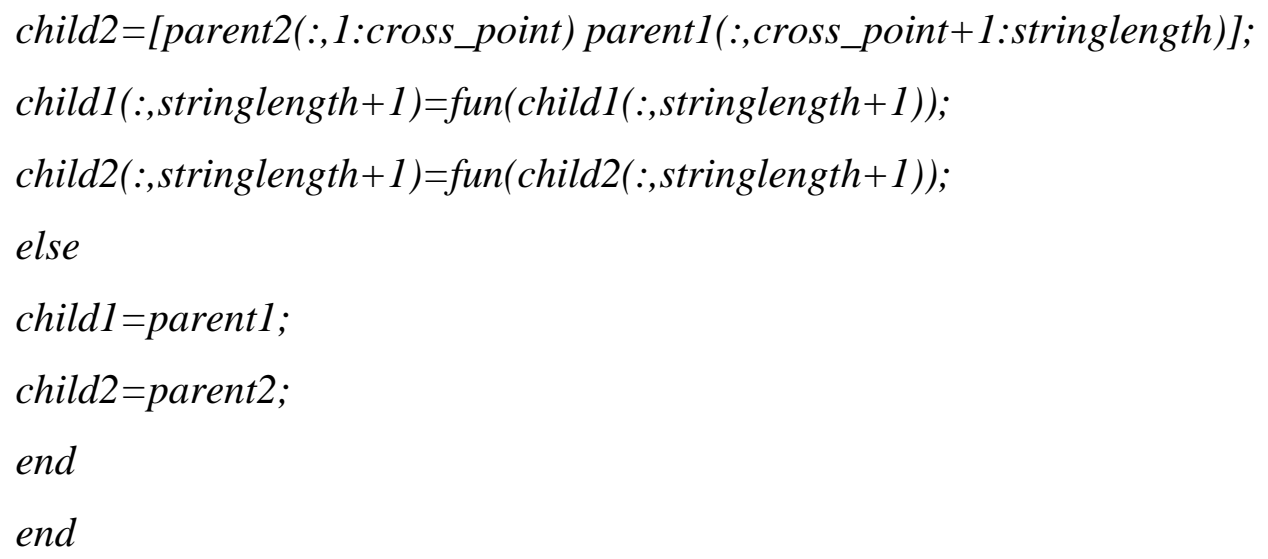

At cross_point, the two chromosomes are split. Then the first part of parent1 and second half of parent 2 forms the first child chromosome. Similarly, the second part of parent 1 and first part of parent 2 forms child2. pc is the probability of crossover. In the case of two-point crossover, two crossover points are selected in each chromosome. The part between the two points is interchanged between the two parents to form two new chromosomes. String length represents the length of each chromosome.

\section{5) Mutation}

Mutation is carried out for the individuals after the crossover procedure. Mutation is the process where only one parent is involved to form a new chromosome. Some random genes are selected for mutation or change. Usually the probability of mutation is chosen to be less than the probability of crossover. Let pm be the probability of mutation. A general algorithm for mutation can be shown as:

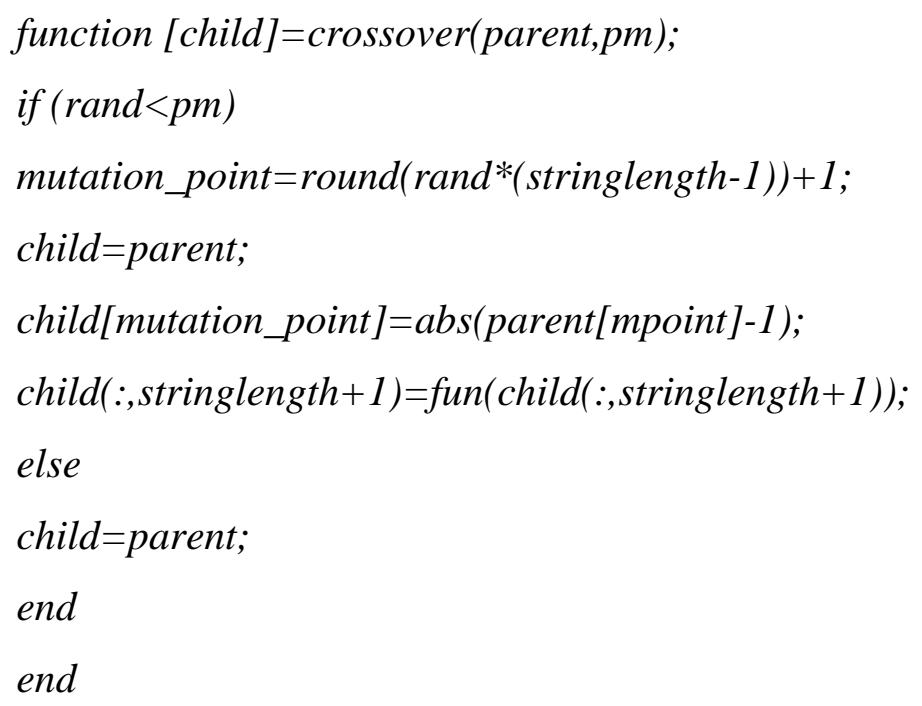


A mutation point, or mutation_point, is selected as the point where mutation occurs. At that point, 0 changes to 1 and vice versa. The fitness value has to be calculated for the new individuals. Thus a new population will be formed, by maintaining the population size.

\section{6) Termination}

These processes take place in an iterative manner. But certain terminating criterions are provided. It could either be the number of iterations, or a particular threshold value that has to be attained, or else the time taken for implementation. When any of these criterion reaches, the iteration automatically stops, and the first chromosome in the current population is selected as the best individual, or as the optimum channel allocation to the problem.

\section{RESULTS OF SIMULATION}

The simulation model for D2D spectrum resource system has been implemented using MATLAB programming language. For simulation simplicity, a MBS system of two CAPs cells has been considered. All randomly deployed CAP users also operate at the same $2 \mathrm{GHz}$ carrier frequency and $5 \mathrm{MHz}$ bandwidth as the central MBS, hence qualified as co-channel deployments. In addition, during random spectrum resource deployments, any two CAPs must be at least 20m apart from each other because each house defines an indoor region with a radius of $10 \mathrm{~m}$. An example of co-channel D2D spectrum resource network deployments from the simulation is shown in Figure 3.

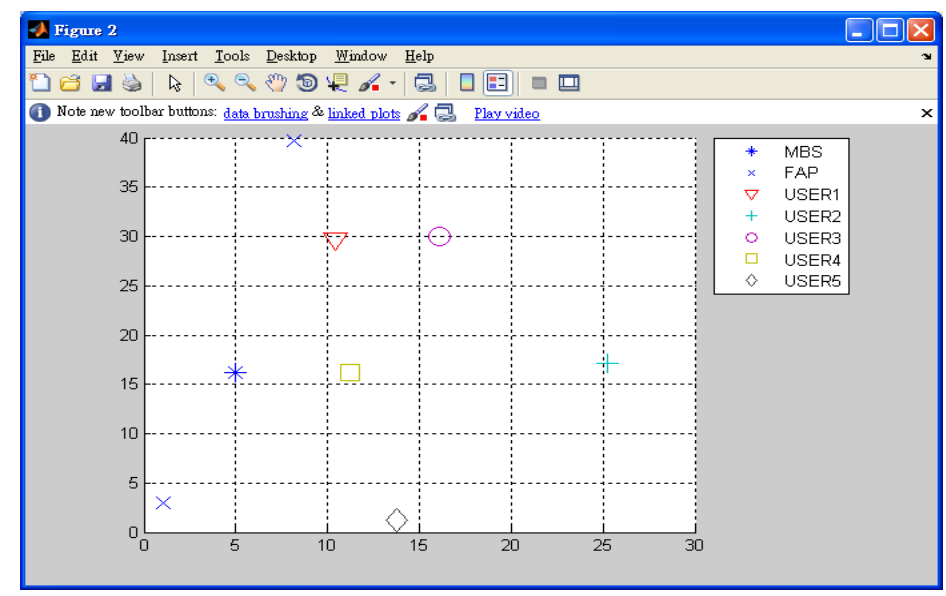

Figure 3 The Spectrum Resource Deployments with Different D2D Users 
All users, both indoors and outdoors, have a spectrum resource identification (i.e. cell_ID) indicating the FAP the user owns (but may not necessary be connected to at the moment), or the FAP the user is allowed access.

After a user has select a FAP to be connected based on the received pilot signal power, that FAP will then execute the access control mechanism to determine whether or not to grant access to the requesting user based on the user classification. Currently, the two widely-recognized access control mechanisms are the Closed access control (CAC) and the Open access control (OAC). Based on different access control mechanisms deployed, a spectrum resource user may experience different treatments from the same FAP.

The performance of the GA algorithm in optimizing SINR D2D spectrum resource network deployments in each timeslot. From Fig. 4 illustrates a series of timeslot of SINR performance in a D2D spectrum resource network.

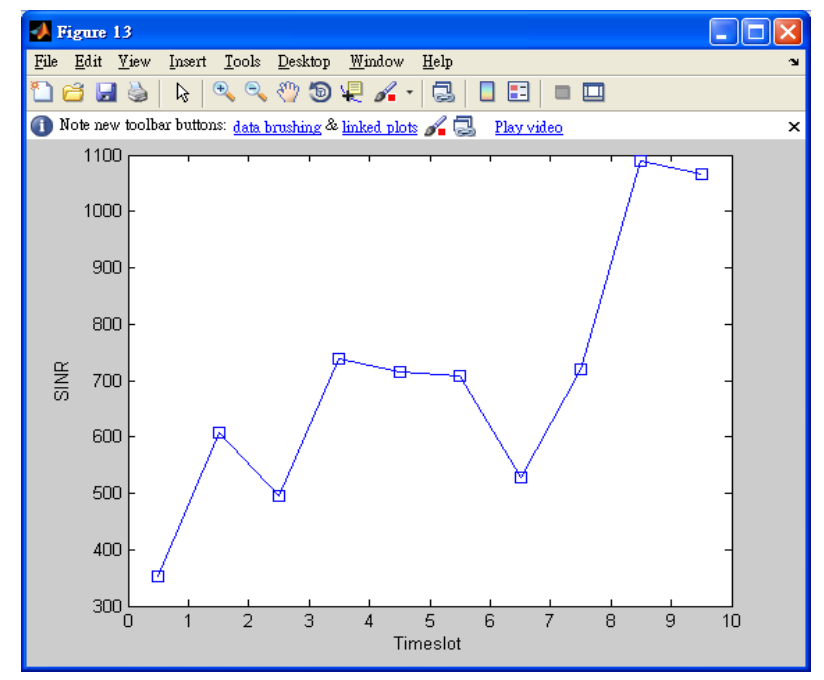

\section{Figure 4 The maximization SINR Performance of D2D Spectrum Resource Network in Each Timeslot}

The Figure 4 presented shows that the optimum value for number of generation is 100 when a co-channel D2D spectrum resource network deployments is considered.

In Fig. 5 how an allocation looks into the D2D spectrum resource network channel resource look. This allocation has been done with 5 CAP users and three channel. The different colour 
rectangles are adjacent users (due to the proximity of the CAP users that transmit data using different channel). Also it's noticed that Channel 2 also link with D2D User 2 and 3

, that's because the long distance between User 2 and User 3 where not able to be interfered each other.

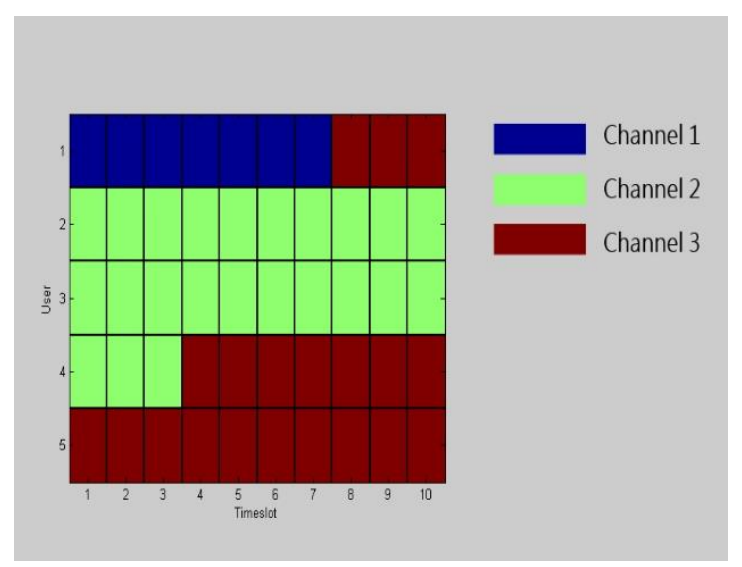

Figure 5 The D2D Channel Allocation

One can also see that the resulting maximum SINR increases pretty consistently with the time varying. This suggests that the algorithm in each run finds a solution that is close to the global maximum. Getting stuck with local maximums would cause inconsistency in the curve. The number of possible allocations is $\mathrm{N}^{\mathrm{F}}$, where $\mathrm{N}$ is the total number of available frequencies, and $\mathrm{F}$ is the number of CAPs in the D2D spectrum resource network. Different scenarios, this is different number of CAPs up to 100 . In the cases studied here this goes from $3^{5}$ to $3^{100}$. Clearly, finding the optimal allocations by going through all the possibilities is difficulty, which justifies the use of more intelligent methods such as genetic algorithms.

\section{CONCLUSIONS}

In this paper, we have discussed some of the challenges of introducing spectrum resources coexistint with current D2D networks. Genetic algorithm also have used to maximization the network performance and channel allocation. From simulations of D2D spectrum resource network, spectrum resource performance, and resource allocation, it was confirmed that the proposed approach. Although the Cognitive access control proposed in this paper is an ideal dynamic resource management mechanism for cellular operators to roll out spectrum resources autonomously, more works should be done to further improve various, such as fair scheduling etc., aspects of the D2D spectrum resource network. 


\section{Reference}

1. V. Chandrasekhar, J. G. Andrews, and A. Gatherer, "Spectrum resource Networks: A Survey", IEEE Communications Magazine, pp 59-67, Sep 2015.

2. D. Lopez-Perez, A.Valcarce, G De La Roche, L.Enjie and Z.Jie, "Access methods to D2D spectrum resources : A downlink system-level case study", The 11th IEEE Singapore International Conference on Communication Systems(Zccs2008),pp. 1 657-1 662, 2014.

3. G. Ganesan and Y. G. Li, "Agility Improvement Through Cooperative Diversity in Cognitive Radio,” in Proc. IEEE Globecom 2005, St. Louis, Missouri, vol. 5, pp. 25052509,December 2015.

4. D. Habibi., H. Zen., I. Ahmad. and J. Wyatt . "Providing QoS for symmetrical voice/video traffic in wireless networks," ICON 2007, 15th IEEE International Conference, vol.10, pp.312-317, 2017.

5. Y.H. Zhang, Cyril Leung, "Resource Allocation in an OFDM-Based Cognitive Radio System ," IEEE Transactions on mobile computing, vol. 57, no. 7, July 2012.

6. E. Hossain and D. Niyato, "Cognitive Radio For Next-generation Wireless Networks: An Approach To Opportunistic Channel Selection In IEEE 802.11-based Wireless Mesh," IEEE JSAC, pp.46-54, vol.16, no.1, February 2009.

7. P. Kyösti, J. Meinilä, L. Hentilä, X. Zhao, T. Jämsä, C. Schneider, M. Narandzić, M. Milojević, A. Hong, J. Ylitalo, Veli-Matti Holappa, M. Alatossava, R. Bultitude, Yvo de Jong, T. Rautiainen ,"WINNER II Channel Models", IST-4-027756 WINNER II D1.1.2 V1.2,2018.

8. K. Kamaluddin, A. Radwan, "Bandwidth Allocation for Handover calls in

9. Mobile Wireless Cellular Networks - Genetic Algorithm Approach", Proceedings of ACIT2010, Dec 14-16 2010. 